\title{
On a New Kinetic Modelling Approach of the Irreversible Quasi-Surface Metallurgical Phase Transformations
}

\author{
Grégory Antoni \\ Haute Ecole d'Ingénierie et de Gestion du Canton de Vaud, Institut COMATEC, Route de Cheseaux 1, \\ 1401 Yverdon-les-Bains, Switzerland \\ Correspondence should be addressed to Grégory Antoni; antoni.gregory@yahoo.fr
}

Received 21 February 2014; Accepted 22 June 2014; Published 20 July 2014

Academic Editor: Kazuhiko Endo

Copyright (C) 2014 Grégory Antoni. This is an open access article distributed under the Creative Commons Attribution License, which permits unrestricted use, distribution, and reproduction in any medium, provided the original work is properly cited.

Irreversible quasi-surface metallurgical phase transformations are the specific response of some metallic materials-such as metals and alloys-subjected to high thermomechanical loads applied very near their surface during the manufacturing processes or after being put into operation. These solid/solid phase transformations can be observed, for example, on the tread of many rails in railroad networks frequented by freight trains. The severe thermal and mechanical loads imposed on the surface of the rails and in the immediate vicinity of the surface by the wheel/rail contact often result in highly localized irreversible metallurgical transformations. A new kinetic model based on a previous study is presented here, which accounts more realistically for the nucleation and growth of these irreversible solid/solid phase transformations resulting from high thermomechanical loads. This metallurgical behavioral model was developed in the framework of continuum thermodynamics with gradients of temperature and internal variables.

\section{Introduction}

The irreversible quasi-surface solid/solid phase transformations observed in many cases in the real industrial settings such as metal forming processes and the subsequent operating phases are the first material responses to high thermomechanical loads. In metallurgical phase transformations of this kind, which often occur in the rails of straight railway sections $[1,2]$ and those frequented by heavy freight trains, the ferrite/pearlite phase is directly transformed into a martensite phase [3]. To account for this process, it does not suffice to take only the thermal history of the material into account like in the standard metallurgical phase transformations [4-6], but both the thermal and the mechanical histories have to be taken into consideration when modelling these irreversible metallurgical transformations because high combined thermomechanical loads are engendered in the wheel-rail contact area, in the presence of strong normal and tangential stresses possibility in addition to a significant increase in the temperature due to the friction process occurring in the contact area [7-10]. In a previous study [11], a kinetic model was presented and discussed for predicting the onset and development of these irreversible quasi-surface solid/solid transformations in the materials subjected to high localized thermomechanical loads applied near the surface. In order to account more accurately, the effects of these high localized thermomechanical loads in the material, a new metallurgical behavioral model, was developed here in the framework of continuum thermodynamics with gradients of temperature and internal variables [12-14].

\section{Kinetic Modelling of the Irreversible Quasi-Surface Metallurgical Phase Transformations: A Local and Nonlocal Approach}

2.1. General Formulation and Constraints in the Framework of Classical Continuum Thermodynamics and Mechanics. The behavioural laws (or constitutive relations) established in the framework of classical continuum thermodynamics [15] for modelling either a fluid or solid media generally have to comply with the following basic physical axioms [16-18]:

(i) the "principle of determinism" (which has also been called the principle of "causality"), which states that knowledge of the past state suffices to be able to determine 
the current state of the material — the current thermodynamic state of an element of matter is only conditioned by its history; that is, its future state cannot influence its current state;

(ii) the "principle of local action" (which has also been called the principle of "local state"), whereby the behaviour of the material at a given point depends only on the physical state variables defined at this material point and not on those of neighboring material points-the thermodynamic state of the element of matter depends only on the history of a set of relevant variables;

(iii) the "principle of objectivity" (which has also been called the principle of "material frame indifference"), which states that the behavioural law must be independent of the observer, meaning that only objective physical quantities can be explicitly taken into account in the behavioral equations in order to ensure the invariance when making a change of the referential frame-the behavioural laws must therefore be written using the objective quantities, that is, the tensorial relations.

According to the above principles, the constitutive laws can be written in the following general form:

$$
\begin{gathered}
\Phi_{i}(X, t)=\underset{-\infty \leq \tau \leq t}{\mathscr{F}_{i}}\left\{\Psi_{j}(X, \tau)\right\}, \\
\text { with } i=1, \ldots, N, \quad j=1, \ldots, M,
\end{gathered}
$$

where $\Phi_{i}$ and $F_{i}(i=1, \ldots, N)$ denote the "response variable" at time $t$ at the material point $X$ and the "functional-memory," associated with the $N$-constitutive relations, and $\Psi_{j}(j=$ $1, \ldots, M)$ are the relevant $M$-physical variables at time $t$ and at the material point $X$, involved in the $N$-constitutive relations.

In addition to the principles presented above, classical continuum mechanics $[17,18]$ also assumes

(iv) the "principle of material simplicity" (which has also been called the principle of "simple material") which stipulates that the state of a material point depends entirely on both the history of the first strain gradient and that of the temperature.

Therefore, (1) can be rewritten thus as

$$
\begin{array}{r}
\Phi_{i}(X, t)=\underset{\substack{\mathscr{F}_{i} \\
-\infty \leq \tau \leq t}}{\mathbf{E}(X, \tau), T(X, \tau)\},} \\
\text { with } i=1, \ldots, N, \quad j=2,
\end{array}
$$

where $\Psi_{1}=$ E and $\Psi_{2}=T$ are the Green-Lagrange strain tensor and the absolute temperature at time $t$, respectively, at the material point $X$. In the case of a nonlinear solid, for example, the behavioural law reads $\mathbf{S}(X, t)=\Phi_{1}(X, t)=$ $\mathbf{S}(\mathbf{E}(X, t), T(X, t))$ where $\mathbf{S}$ is the second Piola-Kirchhoff stress tensor.

In the framework of continuum thermodynamics with internal state variables $[15,19]$, the functional $\mathscr{F}_{i}$ can be replaced by the memory effects of a set of additional internal variables [20] and within the framework of the small perturbations theory (where the initial and current configurations are quasi-identical, i.e., $\Omega^{0} \ni X \equiv x \in \Omega^{t}$ where $x$ is the position of the material point at time $t$ occupying the position $X$ at time $t=0),(2)$ reduces to

$$
\begin{array}{r}
\dot{\Phi}_{i}(t)=F_{i}(\dot{\boldsymbol{\epsilon}}(t), \dot{T}(t), \underbrace{\boldsymbol{\epsilon}(t), T(t), \boldsymbol{\sigma}(t), \boldsymbol{\xi}_{k}(t)}_{=\mathcal{S}}), \\
\text { with } i=1, \ldots, N, \quad k=1, \ldots, P,
\end{array}
$$

where $F_{i}(i=1, \ldots, N)$ denote the functions associated with the $N$-constitutive relations and $\delta$ are the thermomechanical state of the material point at time $t, \sigma$ is the Cauchy stress tensor (where $\boldsymbol{\sigma} \cong \mathbf{S}$ ), $\boldsymbol{\epsilon}$ is the small-strain tensor (where $\boldsymbol{\epsilon} \cong \mathbf{E}$, after linearizing of the operator), and $\boldsymbol{\xi}_{k}(k=1, \ldots, P)$ are the internal state variables in finite number $P$ (which can be arbitrary order tensors including the scalars and vectors), respectively. The set $(\boldsymbol{\epsilon}, T)$ are the "driving" variables responsible for the thermodynamic process (they have also been called "observable" state variables) and the laws of evolution associated with these variables $(\dot{T}, \dot{\boldsymbol{\epsilon}})$ can be of any kind, a priori [21]. It is worth noting that along with the assumption of the small perturbations a tensor of an arbitrary order $\mathbf{r}$ satisfies $\mathbf{r}(X, t) \cong \mathbf{r}(x, t)$ and $\left(\nabla_{X} \mathbf{r}(t)\right)(X) \cong$ $\left(\nabla_{x} \mathbf{r}(t)\right)(x)$.

In the case of a classical problem in continuum mechanics, (3) can be rewritten in the following form:

$$
\begin{aligned}
\dot{\Phi}_{1}(t)=\dot{\boldsymbol{\sigma}}(t)= & F_{1}\left(\dot{\boldsymbol{\epsilon}}(t), \dot{T}(t), \boldsymbol{\epsilon}(t), T(t), \boldsymbol{\sigma}(t), \boldsymbol{\xi}_{k}(t)\right), \\
\dot{\Phi}_{l}(t)=\dot{\boldsymbol{\xi}}_{k}(t)= & F_{l}\left(\dot{\boldsymbol{\epsilon}}(t), \dot{T}(t), \boldsymbol{\epsilon}(t), T(t), \boldsymbol{\sigma}(t), \boldsymbol{\xi}_{k}(t)\right), \\
& \quad \text { with } l=2, \ldots,(P+1), \quad k=1, \ldots, P .
\end{aligned}
$$

Note that in (3) and (4) the position of the material point $X$ has been omitted for the sake of simplicity in the mathematical notations.

2.2. Local Approach: A First Model. In line with (2), a local model was proposed in a previous study to describe these irreversible near-surface metallurgical transformations [11]:

$$
z(X, t)=\underset{-\infty \leq \tau \leq t}{\mathscr{H}}\{\boldsymbol{\epsilon}(X, \tau), T(X, \tau)\}
$$

In addition, (5) can be rewritten as a kinetic law (see (4)b):

$$
\dot{z}=f(\dot{\boldsymbol{\epsilon}}, \dot{T}, \mathcal{S}), \quad \text { with } \mathcal{S}=\{\boldsymbol{\sigma}, \boldsymbol{\epsilon}, T, z\},
$$

where $z \in[0,1]$ is the metallurgical variable (and $z=\xi_{1}$ is the only internal variable and moreover it is a scalar).

Comments. (i) Initially, nontransformed material point $(z=$ 0 ) is composed of a full ferrite/pearlite phase; then when the partial transformation $(z \in] 0,1[$ ) occurs, this material point is composed of a mixture of two phases (a ferrite/pearlite and a martensite phase), and when the transformation has been completed $(z=1)$, a full martensite phase is present.

(ii) The kinetics of irreversible near-surface solid/solid phase transformations depend on both the temperature and the strain history of the material (see (5)). A schematic 


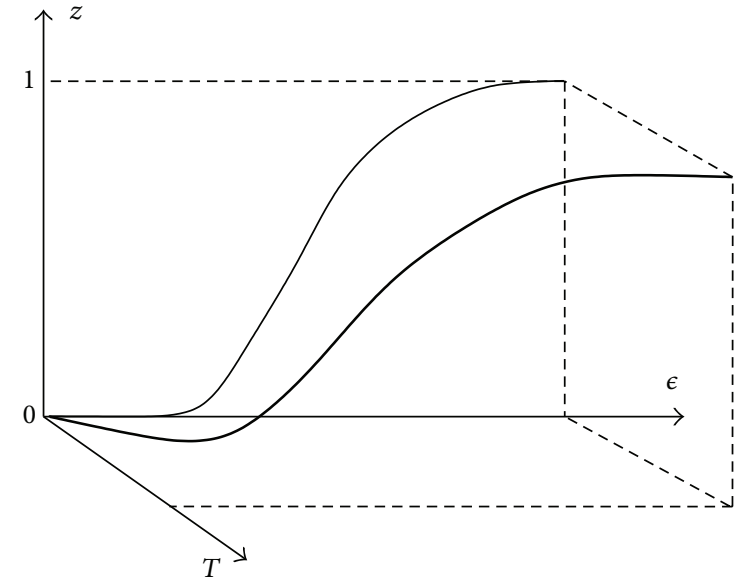

FIGURE 1: Schematic diagram of the metallurgical variable $z$ in function of the strain and temperature variables $(\epsilon, T)$.

diagram of the metallurgical variable $z$ is presented in Figure 1.

(iii) The thermodynamic admissibility of models of this kind was discussed and tested in [10, 22].

(iv) Some numerical results of this local model were shown in the case of a one-dimensional problem [11]. In line with the obtained results, the proposed local model is able to predict the nucleation and growth of these irreversible metallurgical phase transformations near the surface where high thermomechanical loads are applied. However, even if this model may describe a transformed maximum layer which is quite realistic, it cannot take into account the possible existence of strong mechanical and thermal gradients in the material under high thermomechanical loads.

In order to improve the prediction of these irreversible metallurgical transformations occurring in the presence of high localized thermomechanical loads near the surface of the material, a new kinetic model is presented in Section 2.3.

\subsection{Nonlocal Approach: Gradient Modelling with the Tem-} perature and Internal State Variables. For several years, the nonlocal theories developed in the framework of continuum mechanics [23] have been developed because classical continuum theory cannot be used to predict some phenomena involved in the common mechanical problems such as the size effects $[24,25]$ or the localization of strain and damage in some nonlinear solid materials as metals, rocks, and concretes [26]. Since the local action (ii) and the material simplicity (iv) (see Section 2.1) are not necessarily satisfied and their relevance is therefore questionable [27], the nonlocal continuum theory can be used to integrate the weighted averages [28] and higher-order gradients of state variables, such as the observable variables (strain, temperature) or internal variables (characteristic of the dissipative processes under consideration) [12, 29]. Gradient theories are therefore widely used to model the localization of the strain and damage in a large class of solid materials [30-39].
In the framework of gradient theory, (1) can be rewritten as follows:

$$
\begin{aligned}
\Phi_{i}(X, t) & =\underset{\substack{-\infty \leq \tau \leq t \\
-\infty}}{\widetilde{F}_{i}}\left\{\Psi_{j}(X, \tau), \frac{\partial^{n} \Psi_{j}}{\partial X^{n}}(X, \tau)\right\}, \\
\text { with } i & =1, \ldots, N, \quad j=1, \ldots, M, \quad n>0,
\end{aligned}
$$

where $\Phi_{i}$ and $\widetilde{\mathscr{F}}_{i}(i=1, \ldots, N)$ denote the "response variable" at time $t$ at the material point $X$ and the "functional-memory," associated with the $N$-constitutive relations, depending on $\Psi_{j}$ and $\partial^{n} \Psi_{j} / \partial X^{n}(j=1, \ldots, M$ and $n>0$ ) which are the relevant physical variables and their $n$ order gradients involved in the latter, respectively.

In order to account for the possible existence of strong mechanical and thermal gradients in the material subjected to high thermomechanical loads, the laws of evolution can be written (assuming the presence of small perturbations) as follows:

$$
\begin{aligned}
& \dot{\Phi}_{i}(t) \\
& =\widetilde{F}_{i}\left(\dot{\boldsymbol{\epsilon}}(t), \dot{T}(t), \boldsymbol{\epsilon}(t), T(t), \boldsymbol{\sigma}(t), \boldsymbol{\xi}_{k}(t), \nabla^{n} \boldsymbol{\xi}_{k}(t), \nabla T(t)\right), \\
& \text { with } i=1, \ldots, N, \quad k=1, . ., P, \quad n>0,
\end{aligned}
$$

where $\nabla$ and $\nabla^{n}$ denote the first gradient and the $n$-order gradient operator, respectively. It should be noted that only the first temperature gradient $\nabla T$ and higher-order gradients associated with the internal state variables $\nabla^{n} \boldsymbol{\xi}_{k}\left(n \in \mathbb{N}^{*}\right)$ are used here in the modelling procedure.

In line with (8), we assume that the law of evolution of the metallurgical variable $z=\xi_{1}$ in the irreversible quasi-surface solid/solid phase transformations can be written as

$$
\dot{z}=\tilde{f}\left(\dot{\boldsymbol{\epsilon}}, \dot{T}, \boldsymbol{\epsilon}, T, \boldsymbol{\sigma}, \nabla z, \nabla^{2} z, \nabla T\right),
$$

where $\nabla^{2} \equiv \Delta$ denotes the Laplace operator (or Laplacian). Note that in this gradient model it is assumed that the kinetic law can be a function of the first gradient of the temperature $\nabla T$ as well as the first- and second-order gradient of the metallurgical variable $\left(\nabla z, \nabla^{2} z\right)$. Higher-order gradients of the Laplacian $(n>2)$ are therefore not taken into account here.

The nonlocal kinetic model for describing these irreversible quasi-surface solid/solid phase transformations is

$$
\begin{aligned}
& \dot{z}=\dot{z}(\boldsymbol{\sigma}, z, \nabla z, \Delta z, T, \nabla T) \\
&=\kappa \frac{\langle 1-z\rangle}{\eta}\left(\frac{\langle Y(\boldsymbol{\sigma}, z, \nabla z, \Delta z, T, \nabla T)\rangle}{K}\right)^{m}, \\
& Y(\boldsymbol{\sigma}, z, \nabla z, \Delta z, T, \nabla T)= \gamma \sigma_{v m}+\delta P \\
&-\sigma_{y}(T, \nabla T, z, \nabla z, \Delta z),
\end{aligned}
$$




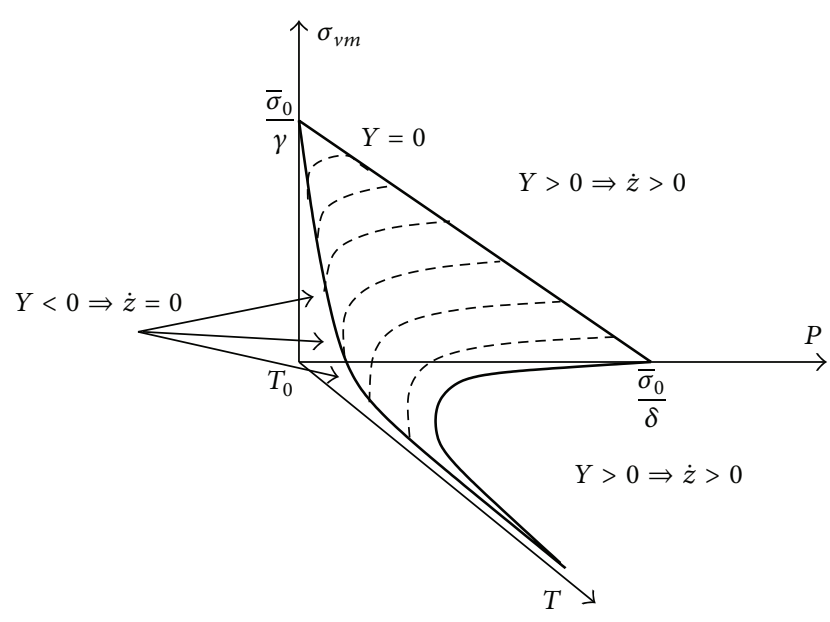

FIGURE 2: Schematic diagram of the yield criterion $Y$ associated with the irreversible quasi-surface solid/solid phase transformation in the plane $\left(\sigma^{v m}, P, T\right)$ with $\sigma_{y}=\sigma_{0}=\bar{\sigma}_{0} \exp \left[-\alpha_{1}\left(T-T_{0}\right) / T_{0}\right]$ and $z=0$ (i.e., $\nabla z=\Delta z=\mathbf{0}$ ).

$$
\begin{aligned}
& \sigma_{y}(T, \nabla T, z, \nabla z, \Delta z)=\sigma_{0}(T, \nabla T)+\beta_{1} z \\
& -\beta_{2}\|\nabla z\|+\beta_{3} \Delta z, \\
& \sigma_{0}(T, \nabla T)=\left\{\begin{array}{c}
\bar{\sigma}_{0} \exp \left(-\alpha_{1} \frac{T-T_{0}}{T_{0}}\right), \\
\text { or } \bar{\sigma}_{0} \exp \left(-\alpha_{2} \frac{\|\nabla T\|}{T_{0}}\right), \\
\text { or } \\
\bar{\sigma}_{0} \exp \left(-\alpha_{1} \frac{T-T_{0}}{T_{0}}\right) \exp \left(-\alpha_{2} \frac{\|\nabla T\|}{T_{0}}\right),
\end{array}\right.
\end{aligned}
$$

where $Y$ denotes the yield function associated with the irreversible solid/solid phase transformation, $\sigma_{y}$ is the current yield strength of the phase transformation, $\bar{\sigma}_{0}$ is the initial yield strength (when $T=T_{0}$ or $\nabla T=\mathbf{0}$ ), $T_{0}$ is the initial temperature, $\sigma_{v m}=\left[3 / 2\left(\sigma^{d}: \sigma^{d}\right)\right]^{1 / 2}$ is the von Mises (equivalent) stress, $-P=(1 / 3) \operatorname{Tr}(\boldsymbol{\sigma})$ and $\boldsymbol{\sigma}^{d}$ are the spherical and deviatoric part of the Cauchy stress tensor $\left(\boldsymbol{\sigma}=-P \mathbf{G}+\boldsymbol{\sigma}^{d}\right.$ where $\mathbf{G}$ is the metric tensor), $\operatorname{Tr}(\cdot)$ is the trace operator, $\kappa$ is a material parameter associated with the solid/ solid phase transformation, $\beta_{1}, \beta_{2}$, and $\beta_{3}$ are the coefficients associated with the local and nonlocal terms of the metallurgical variable $z, \eta$ is the characteristic time of the viscous effects associated with the phase transformation (or the relaxation time), $m$ and $K$ are two coefficients of viscosity associated with the material in question (the viscosity exponent and the resistance coefficient, resp.), $\alpha_{1}$ and $\alpha_{2}$ are the coefficients associated with the local and nonlocal terms of the temperature $T, H(\cdot)$ denotes the Heaviside step function $(H(x)=1$ when $x \geq 0$ and $H(x)=0$ when $x<0),\langle\cdot\rangle$ denotes the Macaulay brackets $(\langle x\rangle=x H(x))$, and $\|\cdot\|$ is the Euclidean norm $\left(\|\mathbf{x}\|=(\mathbf{x} \cdot \mathbf{x})^{1 / 2}\right.$ where $\mathbf{x}$ is a vector and $\cdot$ is the inner product).

Comments. (i) The gradient model presented in (10) belongs to a class of rate-dependent models for the inelastic behavior of materials. The inelastic behaviour investigated here obeys a viscoplastic law of Perzyna type [40, 41]. The convex domain, introduced to account for the irreversibility of solid/solid phase transformations, is limited by the criterion surface equation: $Y=0$ (see Figure 2). The yield criterion $Y$ (11) depends on the stress tensor $(\boldsymbol{\sigma})$, the metallurgical variable $(z)$, the temperature $(T)$, the first gradient of the temperature and that of the metallurgical variable $(\nabla T, \nabla z)$, and the second-order gradient of the metallurgical variable $(\Delta z)$; that is, $Y=Y(\sigma, z, \nabla z, \Delta z, T, \nabla T)$. When the yield criterion satisfies (i) $Y>0$, then $\dot{z}>0$ and the phase transformation increases; (ii) $Y<0$, then $\dot{z}=0$ and the phase transformation remains unchanged.

(ii) The nonstandard metallurgical phase transformations studied here are activated by a certain level of mechanical stresses state, such as normal and shear stresses, via $P$ and $\sigma_{v m}$, possibly accentuated by the temperature $T$ and its gradient $\nabla T$ and also by the metallurgical state via the scalar variable $z$, the first and second gradient of this variable $(\nabla z, \Delta z)$. The current yield strength associated with the phase transformation $\sigma_{y}$ (12) therefore depends on these different local and nonlocal terms; that is, $\sigma_{y}=\sigma_{y}(T, \nabla T, z, \nabla z, \Delta z)$.

(iii) This metallurgical transformation occurs when the mechanical stresses state reaches a sufficient magnitude to trigger the dislocation motion processes in the material caused by the strong strain incompatibilities between the former phase (the ferrite/pearlite phase) and the new phase (the martensite phase). The initiation and development of these solid/solid phase transformations are strongly influenced by the thermal and mechanical state of the material. The initial yield strength associated with the phase transformation $\sigma_{0}$ (13) therefore depends on the temperature of the temperature variable $T$ and its first gradient $\nabla T$; that is, $\sigma_{0}=\sigma_{0}(T, \nabla T)$. The increase in the temperature and/or the temperature gradient can be able to considerably reduce the initial yield strength $\sigma_{0}$ (13). The coefficient $\alpha_{2}$ associated with the nonlocal terms of the temperature $T$ is a characteristic thermal length which serves to determine the active zone in the thermal gradient involved in the metallurgical transformation. Since the local and nonlocal metallurgical states also determine the phase transformation progress, the metallurgical variable $z$ (local term) and the first and second gradient of $z$ (nonlocal terms: $\nabla z, \Delta z$ ) are introduced. In line with [42], a linear coupling of the local and nonlocal terms associated with the metallurgical variable is introduced here (see (12)). During transformations of this kind, a hardening process occurs [7], which is simulated here by the term $\beta_{1} z$ where $\beta_{1}$ denotes the linear isotropic hardening coefficient (which is a dimension of a stress) $[8,10]$. In the nonlocal term $\beta_{2} \nabla z$, an intrinsic characteristic length parameter $l$ can be introduced into the gradient coefficient $\beta_{2}$ so that $\beta_{2}=\bar{\sigma}_{0} l$. Another intrinsic characteristic length parameter $L$ can also be introduced into the nonlocal term $\beta_{3} \Delta z$ where $\beta_{3}=\bar{\sigma}_{0} L^{2}$ (see $[42,43]$ ). The first-order 

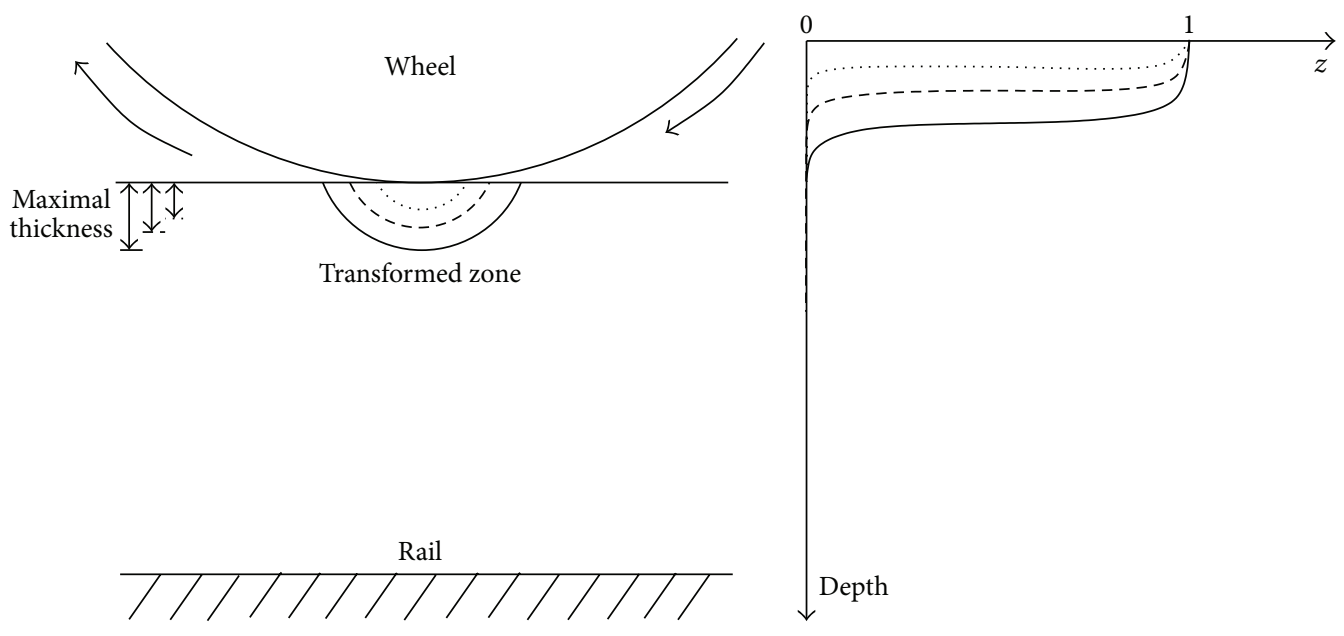

(b)

Figure 3: (a) Schematic diagram of the wheel/rail problem arising in the case of an irreversible quasi-surface metallurgical phase transformation; (b) schematic diagram of the qualitative distribution of the metallurgical variable $z$ in the depth of the rail. The qualitative predictions of $z$-field which will be obtained using a local approach (solid line) and a nonlocal approach (dashed and dotted lines). The dashed line shows the effects of the temperature $(T)$ and its gradient $(\nabla T)$ on the $z$-field. The dotted line gives the effects of the metallurgical nonlocal terms $(\nabla z, \Delta z)$ on the $z$-field.

gradient $\nabla z$ is included here in order to take into account the size effects in these metallurgical transformations. The localization of the transformation is described by the secondorder gradient $\nabla z$ (see $[44,45]$ ).

(iv) The rate-independent plasticity is the limit case of the rate-dependent viscoplastic materials, that is, it occurs when the viscous effects vanish (the relaxation time tends to zero, $\eta \rightarrow 0)$.

(v) The validity domain of the kinetic model is ensured by the term $\langle 1-z\rangle$, that is, when the maximum value of $z$ is reached $z=1$ (in the case of the full phase transformation), then $\dot{z}=0$.

(vi) This nonlocal kinetic model can reduce to a local model in cases where the internal variables $\boldsymbol{\xi}_{k}(k \geq 1)$ remain constant in the space; that is, when $\boldsymbol{\xi}_{k}(\mathbf{x}, t)=\boldsymbol{\xi}_{k}(t)$, then $\nabla \boldsymbol{\xi}_{k}(\mathbf{x}, t)=\Delta \boldsymbol{\xi}_{k}(\mathbf{x}, t)=\mathbf{0}$. If the metallurgical variable $z=\xi_{1}$ satisfies the above relation, then the kinetic law will be of the same type as that presented in Section 2.2.

(vii) The coefficients in the kinetic law under consideration here are the viscosity exponent $m$ (which is dimensionless), the resistance factor $K$ (which is a dimension of a stress), and the relaxation time $\eta$ (which is dimension of a time). These coefficients depend, a priori, on the temperature and on the material in question. In addition, the resistance factor $K$ can be also a function of the set of the internal variables $\xi_{k}$; that is, $K=K\left(T, \boldsymbol{\epsilon}, \boldsymbol{\xi}_{k}\right)$.

(viii) In line with the first kinetic model (Section 2.2), the thermodynamic admissibility of the model developed here can also be confirmed [7, 10, 22] by simply checking whether the Clausius-Duhem inequality is satisfied $[20,21]$.

(ix) In the first step, we can take $m=1$ and $K=\bar{\sigma}_{0}$. In the same way, we can give some parameters such as $\gamma$ (dimensionless), $\delta$ (dimensionless), $\alpha_{1}$ (dimensionless), and a unit value. The values of the parameters, $\beta_{1}$ (a dimension of a stress), $\beta_{2}=\bar{\sigma}_{0} l$ (a dimension of a force per length), $\beta_{3}=\bar{\sigma}_{0} L^{2}$ (a dimension of a force), $\bar{\sigma}_{0}$ (a dimension of a stress), and $\alpha_{2}$ (a dimension of a length), selected have to be determined by performing experimental tests.

(x) The value of the material parameter $\kappa$ depends on the irreversible solid/solid phase transformation investigated here: $\kappa=10^{2}$ (see [22] for more details).

(xi) Figure 3(a) presents the wheel/rail problem arising in the case of an irreversible quasi-surface metallurgical phase transformation occurring in the rail just below the contact zone. The qualitative distribution of the metallurgical variable $z$ in the depth of the rail associated with the proposed kinetic model is described in Figure 3(b), where the predicted $z$ fields using a local approach (solid line) and a nonlocal approach (dashed and dotted lines) are presented. In line with the obtained results in the previous study [11], the effects of the temperature $T$ and its first gradient $\nabla T$ (dashed line) on the $z$-field are also shown qualitatively, along with the effects of the nonlocal metallurgical terms $\nabla z$ and $\Delta z$ (dotted line). It is worth pointing out that the temperature increase in a wheel/rail problem may range from few degrees to hundred degrees Celsius or even more (see [46-49] for more details). The nonlocal kinetic model presented here can therefore account for the high localized thermomechanical fields occurring near and in the immediate vicinity of the contact zone and thus predict more accurately than the previous model the irreversible solid/solid phase transformations occurring just under the rail surface $[8,10]$.

\section{Concluding Remarks}

In this paper, a new kinetic model is presented for predicting the onset and development of the irreversible quasisurface solid/solid phase transformations occurring on the 
tracks of some railways. Based on the gradient theory, this metallurgical behavioral model is extended here to include irreversible solid/solid phase transformations taking into account the possible existence of strong mechanical and thermal gradients in the material as the result of the heavy thermomechanical loads induced by the wheel/rail contacts. For this purpose, the gradients of the temperature and the metallurgical variable have been explicitly included in the model.

\section{Conflict of Interests}

The author declares that there is no conflict of interests regarding the publication of this paper.

\section{Acknowledgment}

The author is indebted to Dr. Jessica Blanc for her help with this paper.

\section{References}

[1] W. Österle, H. Rooch, A. Pyzalla, and L. Wang, "Investigation of white etching layers on rails by optical microscopy, electron microscopy, X-ray and synchroton X-ray diffraction," Materials Science and Engineering A, vol. 303, no. 1-2, pp. 150-157, 2001.

[2] A. Pyzalla, L. Wang, E. Wild, and T. Wroblewski, "Changes in microstructure, texture and residual stresses on the surface of a rail resulting from friction and wear," Wear, vol. 251, no. 2, pp. 901-907, 2001.

[3] G. Baumann, H. J. Fecht, and S. Liebelt, "Formation of whiteetching layers on rail treads," Wear, vol. 191, no. 1-2, pp. 133-140, 1996.

[4] V. Raghavan, Physical Metallurgy: Principles and Practice, Prentice-Hall of India, 2006.

[5] D. A. Porter, K. E. Easterling, and M. Y. Sherif, Phase Transformations in Metals and Alloys, CRC Press, New York, NY, USA, 2009.

[6] E. Pereloma and D. V. Edmonds, Phase Transformations in Steels: Fundamentals and Diffusion-Controlled Transformations, Woodhead, 2012.

[7] G. Antoni, Transformations Tribologiques de Surface: une approche thermo-mécanique, [Ph.D thesis], Université de Provence, Marseille, France, 2010.

[8] G. Antoni, "Contribution to the modelling of the Tribological Surface Transformations," ISRN Tribology, vol. 2013, Article ID 254705, 6 pages, 2013.

[9] G. Antoni, "Assessment of thermomechanical couplings in Tribological Surface Transformations: application to the irreversible near-surface solid-solid phase transformations," ISRN Tribology, vol. 2013, Article ID 525708, p. 6, 2013.

[10] G. Antoni, "A phenomenological modelling with thermomechanical coupling for Tribological Surface Transformations (TSTs)," International Journal of Engineering Science, vol. 78, pp. 218-232, 2014.

[11] G. Antoni, "A kinetic model for Tribological Surface Transformations occuring on the railroads: irreversible near-surface metallurgical transformations," ISRN Metallurgy, vol. 2012, Article ID 590792, 6 pages, 2012.
[12] P. Ireman and Q. Nguyen, "Using the gradients of temperature and internal parameters in Continuum Thermodynamics," Comptes Rendus-Mecanique, vol. 332, no. 4, pp. 249-255, 2004.

[13] Q. S. Nguyen and S. Andrieux, "The non-local generalized standard approach: a consistent gradient theory," Comptes Rendus Mécanique, vol. 333, no. 2, pp. 139-145, 2005.

[14] Q. Nguyen, "Gradient thermodynamics and heat equations," Comptes Rendus - Mecanique, vol. 338, no. 6, pp. 321-326, 2010.

[15] P. Germain, Q. S. Nguyen, and P. Suquet, "Continuum Thermodynamics," Journal of Applied Mechanics, vol. 50, no. 4 b, pp. 1010-1020, 1983.

[16] E. B. Tadmor, R. E. Miller, and R. S. Elliott, Continuum Mechanics and Thermodynamics: From Fundamental Concepts to Governing Equations, Cambridge University Press, 2011.

[17] P. Haupt, Continuum Mechanics and Theory of Materials, Springer, Berlin, Germany, 2002.

[18] I. S. Liu, Continuum Mechanics, Springer, Berlin, Germany, 2002.

[19] B. D. Coleman and M. E. Gurtin, "Thermodynamics with internal state variables," The Journal of Chemical Physics, vol. 47, no. 2, pp. 597-613, 1967.

[20] J. Lemaitre and J. L. Chaboche, Mechanics of Solid Materials, Cambridge University Press, 1994.

[21] J. Garrigues, Fondements de la Mecanique des Milieux Continus, Hermès Science, Paris, France, 2007.

[22] G. Antoni, "Effects of thermomechanical coupling in Tribological Surface Transformations: a one-dimensional modelling including irreversible solid-solid phase transformations and classical plasticity," Journal of Materials, Article ID 892050, 8 pages, 2013.

[23] A. C. Eringen, Nonlocal Continuum Field Theories, Springer, New York, NY, USA, 2002.

[24] E. C. Aifantis, "Strain gradient interpretation of size effects," International Journal of Fracture, vol. 95, no. 1-4, pp. 299-314, 1999.

[25] R. K. Abu Al-Rub and G. Z. Voyiadjis, "A physically based gradient plasticity theory," International Journal of Plasticity, vol. 22, no. 4, pp. 654-684, 2006.

[26] E. C. Aifantis, "On the role of gradients in the localization of deformation and fracture," International Journal of Engineering Science, vol. 30, no. 10, pp. 1279-1299, 1992.

[27] M. Jirasek, "Nonlocal theories in continuum mechanics," Acta Polytechnica, vol. 44, no. 5-6, pp. 16-34, 2004.

[28] Z. P. Bažant and M. Jirásek, "Nonlocal integral formulations of plasticity and damage: survey of progress," Journal of Engineering Mechanics, vol. 128, no. 11, pp. 1119-1149, 2002.

[29] Q. Nguyen, "Variational principles in the theory of gradient plasticity," Comptes Rendus - Mecanique, vol. 339, no. 12, pp. 743-750, 2011.

[30] H. Mühlhaus and E. C. Aifantis, "A variational principle for gradient plasticity," International Journal of Solids and Structures, vol. 28, no. 7, pp. 845-857, 1991.

[31] R. De Borst and H. Muehlhaus, "Gradient-dependent plasticity: formulation and algorithmic aspects," International Journal for Numerical Methods in Engineering, vol. 35, no. 3, pp. 521-539, 1992.

[32] M. Frémond and B. Nedjar, "Damage, gradient of damage and principle of virtual power," International Journal of Solids and Structures, vol. 33, no. 8, pp. 1083-1103, 1996. 
[33] R. de Borst, J. Pamin, and M. G. D. Geers, “On coupled gradientdependent plasticity and damage theories with a view to localization analysis," European Journal of Mechanics A: Solids, vol. 18, no. 6, pp. 939-962, 1999.

[34] E. Lorentz and S. Andrieux, "Variational formulation for nonlocal damage models," International journal of plasticity, vol. 15, no. 2, pp. 119-138, 1999.

[35] B. Nedjar, "Elastoplastic-damage modelling including the gradient of damage: formulation and computational aspects," International Journal of Solids and Structures, vol. 38, no. 30-31, pp. 5421-5451, 2001.

[36] M. Frémond, Non-Smooth Thermomechanics, Springer, New York, NY, USA, 2002.

[37] P. Ireman, A. Klarbring, and N. Strömberg, "A model of damage coupled to wear," International Journal of Solids and Structures, vol. 40, no. 12, pp. 2957-2974, 2003.

[38] J. Zhao, D. Shen, and I. F. Collins, "Thermomechanical formulation of strain gradient plasticity for geomaterials," Journal of Mechanics of Materials and Structures, vol. 1, no. 5, pp. 837-863, 2006.

[39] G. Z. Voyiadjis, G. Pekmezi, and B. Deliktas, "Nonlocal gradient-dependent modeling of plasticity with anisotropic hardening," International Journal of Plasticity, vol. 26, no. 9, pp. 1335-1356, 2010.

[40] P. Perzyna, "Fundamental problems in viscoplasticity," Advances in Applied Mechanics, vol. 9, pp. 243-377, 1966.

[41] P. Perzyna, "Thermodynamic theory of viscoplasticity," Advances in Applied Mechanics, vol. 11, pp. 313-354, 1971.

[42] R. K. Abu Al-Rub and G. Z. Voyiadjis, "Analytical and experimental determination of the material intrinsic length scale of strain gradient plasticity theory from micro- and nanoindentation experiments," International Journal of Plasticity, vol. 20, no. 6, pp. 1139-1182, 2004.

[43] G. Z. Voyiadjis and R. K. A. Al-Rub, "Gradient plasticity theory with a variable length scale parameter," International Journal of Solids and Structures, vol. 42, no. 14, pp. 3998-4029, 2005.

[44] J. Chen, A nonlocal damage model for elasto-plastic materials based on gradient plasticity theory [Ph.D. thesis], Swiss Federal Institute of Technology, Zürich, Switzerland, 2001.

[45] R. A. B. Engelen, Plasticity-induced Damage in Metals-nonlocal modelling at finite strains [Ph.D. thesis], Technische Universiteit Eindhoven, Eindhoven, The Netherlands, 2005.

[46] K. D. Van and M. H. Maitournam, "Thermomechanical state near rolling contact area," Dissipative Processes in Tribology, vol. 27, pp. 423-428, 1994.

[47] K. Dang Van, M. H. Maitournam, and B. Prasil, "Elastoplastic analysis of repeated moving contact application to railways damage phenomena," Wear, vol. 196, no. 1-2, pp. 77-81, 1996.

[48] K. Knothe and S. Liebelt, "Determination of temperatures for sliding contact with applications for wheel-rail systems," Wear, vol. 189, no. 1-2, pp. 91-99, 1995.

[49] M. Ertz and K. Knothe, "A comparison of analytical and numerical methods for the calculation of temperatures in wheel/ rail contact," Wear, vol. 253, no. 3-4, pp. 498-508, 2002. 

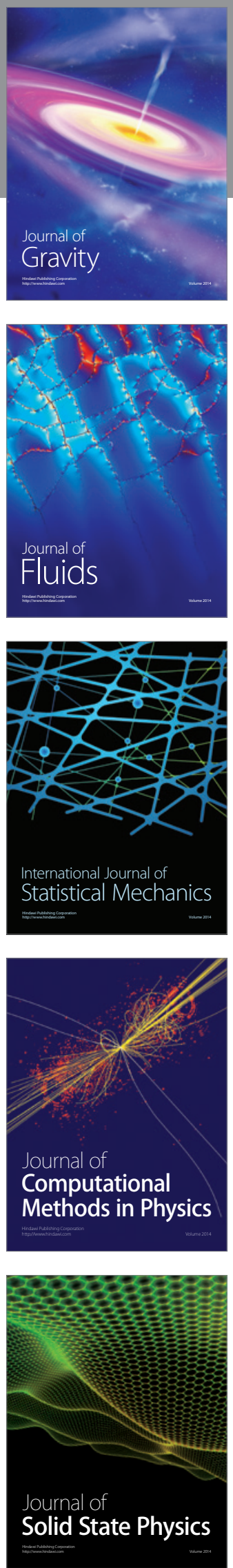

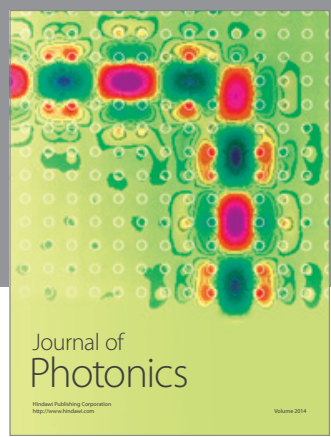

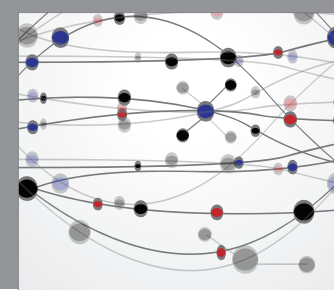

The Scientific World Journal

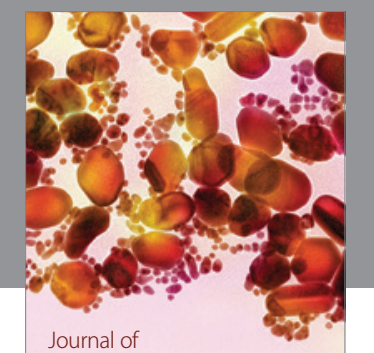

Soft Matter
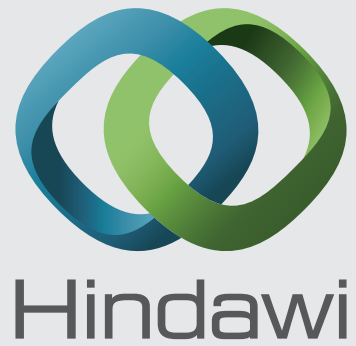

Submit your manuscripts at

http://www.hindawi.com
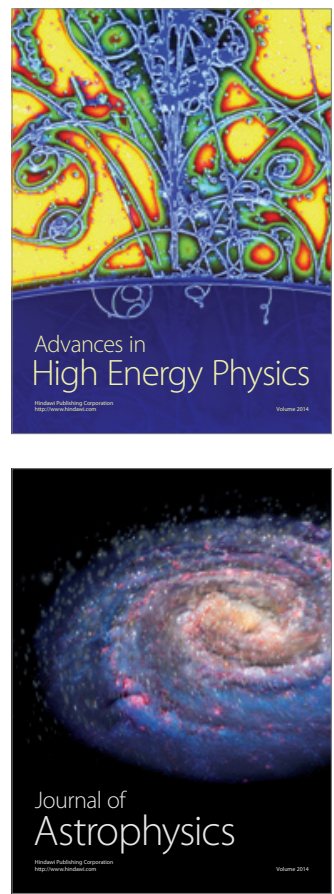
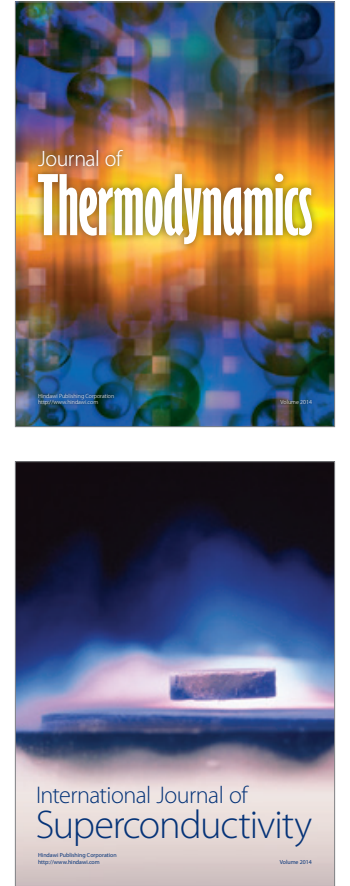
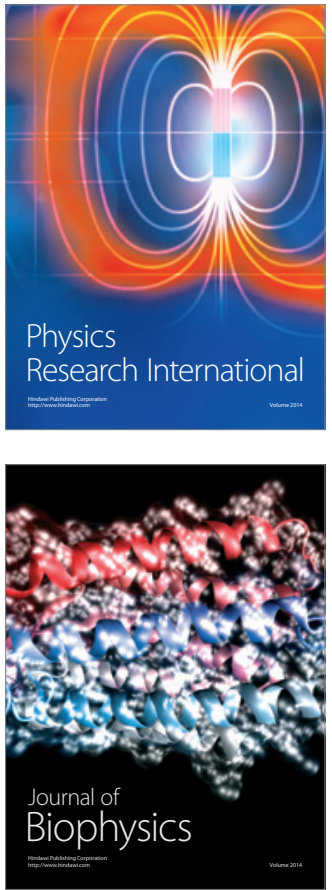
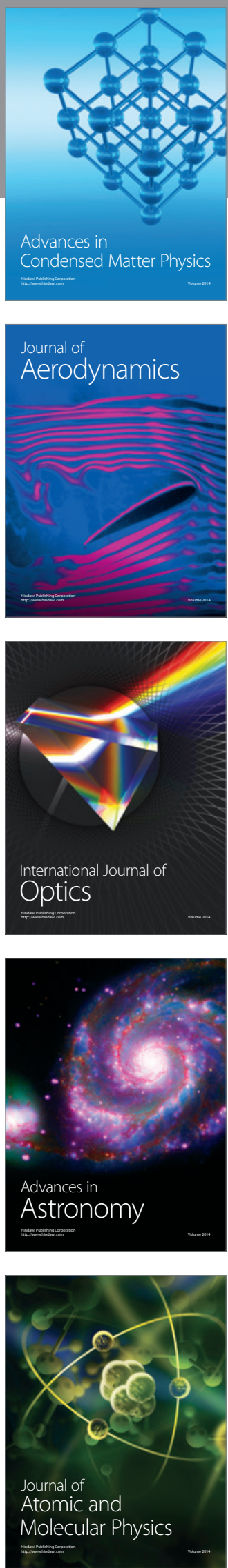